\title{
UNSTABLE PERIODIC WAVE SOLUTIONS OF NERVE AXION DIFFUSION EQUATIONS
}

\author{
RINA LING \\ Depariment of Mathematics and Computer Science \\ California State University, \\ Los Angeles, California 90032 \\ (Received September 9, 1986)
}

ABSTRACT. Unstable periodic solutions of systems of parabolic equations are studied. Special attention is given to the existence and stability of solutions.

REY WORDS AND PHRASES. Periodic wave solutions, Diffusion equations, existence and stability analysis

1980 AMS SUBJECT ClASSIFICATION CODE. $35 \mathrm{k} 40,35 \mathrm{~K} 57$.

\section{INTRODUCTION.}

Diffusion systems of partial differential equations are of great importance in biosciences. In this paper, unstable periodic solutions of systems of the form

$$
\begin{aligned}
& u_{t}=u_{x x}+F(u, w), \\
& w_{t}=G(u, w),
\end{aligned}
$$

are studied. Equations of this type arise in neurophysiology in the study of nerve impulses on nerve axon, see [1,2]. Other classes of diffusion equations are also involved in biology, see for example [3-9].

\section{EXISTENCE OP SOLUTIONS}

It is known that for $G(u, w)=\varepsilon u$, if $\varepsilon>0$ is sufficiently sma11, equation (1.1) has two types of wave solutions, namely, pulse travelling wave solutions and periodic travelling wave solutions. A travelling wave solution is a solution of equation $(1.1)$ of the form

$$
[u(x, t), w(x, t)]=[\phi(z ; c), \psi(x ; c)], z=x+c t
$$

hence $[\phi(z ; c), \psi(z ; c)]$ satisfies the ordinary differential equation

$$
\begin{aligned}
& \frac{d^{2} \phi}{d z^{2}}-c \frac{d \phi}{d z}+F(\phi, \psi)=0, \\
& -c \frac{d \psi}{d z}+G(\phi, \psi)=0 .
\end{aligned}
$$




$$
\lim _{|z| \rightarrow \infty}[\phi(z ; c), \psi(z ; c)]=[0,0]
$$

and a periodic travelling wave solution is a periodic solution of (2.1).

In [10], Evans showed that equation (1.1) has two pulse iravelling solutions with different propagation speeds $c_{1}$ and $c_{2}$. On the existence of periodic travelling wave solutions, Hastings [11] showed that equation (2.1) with $G(u, w)=\varepsilon u$ has a nonconstant periodic solution if $\varepsilon>0$ is sufficiently small and the speed $c$ is limited to a certain range. Rinzel and Keller [12] studied the case in which $F(u, w)$ is a function of $u$ only given by

$$
F(u, w)=\left\{\begin{array}{lll}
u & \text { for } & u \leq a, \\
u-1 & \text { for } & a<u,
\end{array}\right.
$$

where $0<a<1 / 2$. Under this assumption, equation (2.1) has a non-constant periodic solution if $c$ is limited in the range $c_{1}<c<c_{2}$ and the period $p(c)$ is a smooth function of c. They demonstrated the behavior of the function $p(c)$ under the two cases when $a$ is not very small and when a is very sma11. Dai [13] proved the existence and uniqueness of solutions for a general case and studied stability of the solution.

\section{STABILITY ANALYSIS.}

Stability of periodic travelling wave solutions is related to the eigenvalues of a matrix in the following theorem. Let $A(z ; \lambda, c)$ be the matrix

$$
A(z ; \lambda, c)=\left[\begin{array}{ccc}
0 & 1 & 0 \\
\lambda-F_{1}[\phi(z ; c), \psi(z ; c)] & c & -F_{2}[\phi(z ; c), \psi(z ; c)] \\
\frac{G_{1}[\phi(z ; c), \psi(z ; c)]}{c} & 0 & \frac{G_{2}[\phi(z ; c), \psi(z: c)]-\lambda}{c}
\end{array}\right]
$$

where $F_{i}$ and $G_{i}$ denote the partial derivatives as usual, and let $X(z ; \lambda, c)$ be a matrix satisfying the differential equation

$$
\frac{d}{d z} x=A X
$$

with the initial condition $X(0 ; \lambda, c)=I$.

THEOREM 3.1. Suppose the functions $F$ and $G$ in equation (1.1) staisfy (a) $F(0,0)=0$, (b) $G(0,0)=0$ and (c) the matrix $X(p(c) ; \lambda, c)$ has an eigenvalue of modulus 1 , for some complex number $\lambda$ with $\operatorname{Re} \lambda>0$, then a periodic travelling wave solution $[\phi(z ; c), \psi(z ; c)]$ is unstable.

PROOF. With the change of variables,

$$
\begin{aligned}
& z=x+c t, \\
& t=t, \\
& {[u(x, t), w(x, t)]=[\bar{u}(z, t), \bar{w}(z, t)],}
\end{aligned}
$$


equation (1.1) becomes

$$
\begin{aligned}
& \bar{u}_{t}=\bar{u}_{z z}-c \bar{u}_{z}+F(\bar{u}, \bar{w}), \\
& \bar{w}_{t}=-c \bar{w}_{z}+G(\bar{u}, \bar{w}) .
\end{aligned}
$$

The linearized perturbation equation of the above system with respect to the solution $[\phi(z ; c), \psi(z ; c)]$ is

$$
\begin{aligned}
& \overline{\mathrm{U}}_{\mathrm{t}}=\overline{\mathrm{U}}_{z z}-\mathrm{c} \overline{\mathrm{U}}_{\mathrm{z}}+\mathrm{F}_{1}[\phi, \psi] \overrightarrow{\mathrm{U}}+\mathrm{F}_{2}[\phi, \psi] \overline{\mathrm{W}}, \\
& \overline{\mathrm{W}}_{t}=-\mathrm{c} \overline{\mathrm{W}}_{z}+\mathrm{G}_{1}[\phi, \psi] \overline{\mathrm{U}}+\mathrm{G}_{2}[\phi, \psi] \overline{\mathrm{W}},
\end{aligned}
$$

where $\phi=\phi(z ; c)$ and $\psi=\psi(z ; c)$, since $F(0,0)=G(0,0)=0$. Equation (3.2) has a solution of the form

$$
\begin{aligned}
& \vec{U}(z, t)=e^{\lambda t} y_{1}(z ; \lambda), \\
& \vec{W}(z, t)=e^{\lambda t} y_{2}(z ; \lambda),
\end{aligned}
$$

where $\left(y_{1}, y_{2}\right)$ satisfies the following system of linear ordinary differential equations

$$
\begin{aligned}
& \lambda y_{1}=\frac{d^{2} y_{1}}{d z^{2}}-c \frac{d y_{1}}{d z}+F_{1}[\phi, \psi] y_{1}+F_{2}[\phi, \psi] y_{2}, \\
& \lambda y_{2}=-c \frac{d y_{2}}{d z}+G_{1}[\phi, \psi] y_{1}+G_{2}[\phi, \psi] y_{2},
\end{aligned}
$$

where $\phi=\phi(z ; c)$ and $\psi=\psi(z ; c)$. Note that if equation (3.3) has a solution which is bounded for all $z$ in $(-\infty, \infty)$ for a number $\lambda$ with $\operatorname{Re}(\lambda)>0$, then equation (3.2) has a solution $[\bar{U}(z, t), \bar{W}(z, t)]$ which grows exponentially, and hence, the travelling wave solution $[\phi(z ; c), \psi(z ; c)]$ is unstable.

Using Floquet's theory, we can show that equation (3.3) has a bounded non-trivial solution if and only if one of the eigenvalues of $x(p(c) ; \lambda, c)$ is a modulus 1 . Equation (3.3) can be rewritten as

$$
\begin{aligned}
& \frac{d}{d z}\left(\frac{d y_{1}}{d z}\right)=\left(\lambda-F_{1}[\phi, \psi]\right) y_{1}+c \frac{d y_{1}}{d z}-F_{2}[\phi, \psi] y_{2}, \\
& c \frac{d y_{2}}{d z}=G_{1}[\phi, \psi] y_{1}+\left(G_{2}[\phi, \psi]-\lambda\right) y_{2},
\end{aligned}
$$

and so can be represented by the matrix differential equation

$$
\frac{\mathrm{d}}{\mathrm{d} z} \underline{\mathrm{v}}=\mathrm{A}(\mathrm{z} ; \lambda, \mathrm{c}) \underline{\mathrm{v}},
$$


where

$$
\underline{v}=\left[\begin{array}{l}
\mathrm{y}_{1} \\
\frac{\mathrm{dy}_{1}}{\mathrm{dz}} \\
\mathrm{y}_{2}
\end{array}\right]
$$

and the matrix $A$ is as defined before. Now, since the coefficient matrix $A(z ; \lambda, c)$ is a $p(c)$-periodic function of $z$, Floquet's theory yields that equation (3.3) has a bounded non-trivial solution if and only if one of the eigenvalues of the matrix $\mathrm{X}(\mathrm{p}(\mathrm{c}) ; \lambda, c)$ defined before is of modulus 1 . The proof is now complete.

In the following lemma, it is shown that under the special case $\lambda=0$, one eigenvalue of $X(p(c) ; 0, c)$ is unity and the product of the other two eigenvalues is greater than one.

LEMMA 3.1. Suppose (a) $G_{2}(u, w) \geq 0$ for all $u$ and $w$ and (b) $\lambda=0$, let $\mu_{i}(\lambda, c), i=1,2,3$, denote the eigenvalues of $x(p(c) ; \lambda, c)$, then one eigenvalue, say

and

$$
\mu_{1}(0, c)=1
$$

$$
\mu_{2}(0, c) \mu_{3}(0, c)>1
$$

PROOF. Differentiation of equation (2.1) leads to

$$
\begin{aligned}
& \frac{d}{d z}\left(\frac{d^{2} \phi}{d z^{2}}\right)=c \frac{d}{d z}\left(\frac{d \phi}{d z}\right)-F_{1}[\phi, \psi] \frac{d \phi}{d z}-F_{2}[\phi, \psi] \frac{d \psi}{d z} \\
& c \frac{d}{d z}\left(\frac{d \psi}{d z}\right)=G_{1}[\phi, \psi] \frac{d \phi}{d z}+G_{2}[\phi, \psi] \frac{d \psi}{d z},
\end{aligned}
$$

where $\phi=\phi(z ; c)$ and $\psi=\psi(z ; c)$. Therefore the vector

$$
\underline{W}=\left[\begin{array}{c}
\phi \\
\frac{d \phi}{d z} \\
\psi
\end{array}\right]
$$

satisfies the matrix equation

$$
\frac{\mathrm{d}}{\mathrm{dz}} \underline{w_{z}}=A(z ; 0, c) \underline{w_{z}},
$$

that is,

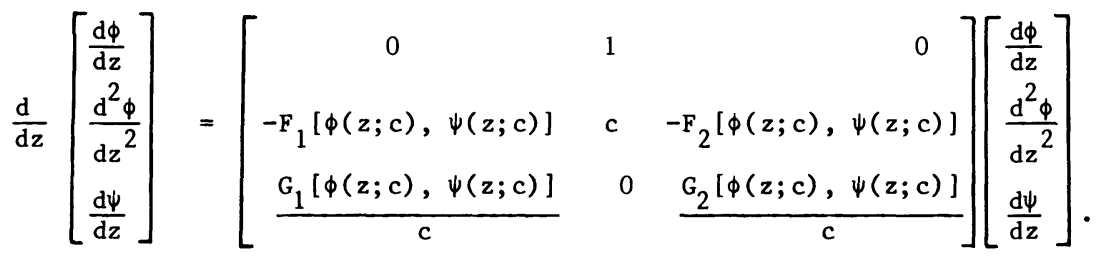


We know that (see for example, Sanchez)

$$
\underline{w_{z}}(z ; c)=X(z ; 0, c) \underline{w_{z}}(0 ; c)
$$

and since $w_{\underline{z}}(z ; c)$ is a $p(c)$ - periodic function of $z$, it follows that

$$
\underline{w_{z}}(0 ; c)=\underline{w_{z}}(p(c) ; c)=X(p(c) ; 0, c) \underline{w_{z}}(0 ; c) .
$$

Thus there is an eigenvalue, say

$$
\mu_{1}(0, c)=1
$$

Further, by Jacobi's formula,

$$
\begin{aligned}
\operatorname{det}\{x(z ; \lambda, c)\} & =\{\operatorname{det} x(0 ; \lambda, c)\} \exp \int_{0}^{z} \operatorname{tr}\{A(\xi ; \lambda, c)\} d \xi \\
& =(1) \exp \int_{0}^{z}\left(c+\frac{G_{2}[\phi, \psi]-\lambda}{c}\right) d \xi
\end{aligned}
$$

In particular,

$$
\begin{aligned}
\operatorname{det}\{x(p(c) ; 0, c)\} & =\exp [c p(c)] \exp \int_{0}^{p(c)} \frac{G_{2}[\phi, \psi]}{c} d \xi \\
& >1
\end{aligned}
$$

since $c>0, p(c)>0$ and $G_{2}(u, w) \geq 0$ for all u,w.

But $\operatorname{det}\{x(p(c) ; 0, c)\}=\mu_{1}(0, c) \mu_{2}(0, c) \mu_{3}(0, c)$ and

$$
\mu_{1}(0, c)=1 \text {, hence } \mu_{2}(0, c) \mu_{3}(0, c)>1 \text {. }
$$

Note that under the assumptions of Lemma 3.1 , either $\left|\mu_{2}(\lambda, c)\right|>1$ or $\mid \mu_{3}(\lambda, c \mid>1$ for $\lambda$ sufficiently sma11. In the next theorem, we will see that if $L(c)$ is decreasing, i.e. $L^{\prime}(c)<0$, then $\mu_{1}(\lambda, c)$ is increasing at $\lambda=0$, i.e. $\left.\frac{\partial}{\partial \lambda} \mu_{1}(\lambda, c)\right|_{\lambda=0}>0$.

THEOREM 3.2. Suppose (a) $\mathrm{p}^{\prime}(\mathrm{c})<0$, then $\left.\frac{\partial}{\partial \lambda} \mu_{1}(\lambda, \mathrm{c})\right|_{\lambda=0}>0$, and hence if (b) the assumptions in Lemma 3.1 also hold, then $\mu_{1}(\lambda, c)>1$ for $\lambda$ sufficiently small.

PROOF: We claim that the following equality

$$
\left.\frac{\partial}{\partial \lambda} \mu_{1}(\lambda, c)\right|_{\lambda=0}=-p^{\prime}(c)
$$

actually holds. 
Recall the vector $\underline{w}(z ; c)$, namely,

$$
\underline{w}=\left[\begin{array}{c}
\phi \\
\frac{d \phi}{d z} \\
\psi
\end{array}\right]
$$

which satisfies the periodicity

$$
\underline{w}(p(c) ; c)=\underline{w}(0 ; c) \text {. }
$$

Differentiation of the above equation with respect to $c$ leads to

$$
\underline{w_{z}}(p(c) ; c) p^{\prime}(c)+\underline{w_{c}}(p(c) ; c)=\underline{w_{c}}(0 ; c) .
$$

Let $\underline{\mathrm{v}}=\underline{\mathrm{v}}^{*}=\left[\mathrm{y}_{1}^{*}(\mathrm{z} ; \lambda, \mathrm{c}), \mathrm{y}_{2}^{*}(\mathrm{z} ; \lambda, \mathrm{c})\right]$ be a solution of equation $(3.3)$ satisfying the initial condition

$$
\underline{\mathrm{v}}(0 ; \lambda, \mathrm{c})=\underline{\mathrm{w}_{z}}(0 ; \mathrm{c})+\lambda \underline{\mathrm{w}_{\mathrm{c}}}(0 ; \mathrm{c})
$$

where $\mathrm{v}(z ; \lambda, c)$ is the vector defined before. We have observed before that $\left[\frac{d \phi}{d z}(z ; c)\right.$, $\left.\frac{\mathrm{d} \psi}{\mathrm{d} z}\right](z ; c)$, which satisfies equation $(3.4)$, is a solution of equation (3.3) under $\lambda=0$. In view of the condition (3.7) and by uniqueness of solutions, we have

$$
\underline{\mathrm{v}}^{*}(\mathrm{z} ; 0, \mathrm{c})=\underline{\mathrm{w}_{\mathrm{z}}}(\mathrm{z} ; \mathrm{c}) \text {. }
$$

Differentiation of equation (3.3) with respect to $\lambda$ leads to

$$
\begin{aligned}
& \mathrm{y}_{1}+\lambda \frac{\partial \mathrm{y}_{1}}{\partial \lambda}=\frac{\mathrm{d}^{2}}{\mathrm{dz}^{2}}\left(\frac{\partial \mathrm{y}_{1}}{\partial \lambda}\right)-\mathrm{c} \frac{\mathrm{d}}{\mathrm{dz}}\left(\frac{\partial \mathrm{y}_{1}}{\partial \lambda}\right)+\mathrm{F}_{1}[\phi, \psi] \frac{\partial \mathrm{y}_{1}}{\partial \lambda}+\mathrm{F}_{2}[\phi, \psi] \frac{\partial \mathrm{y}_{2}}{\partial \lambda} \\
& \mathrm{y}_{2}+\lambda \frac{\partial \mathrm{y}_{2}}{\partial \lambda}=-\mathrm{c} \frac{\mathrm{d}}{\mathrm{dz}}\left(\frac{\partial \mathrm{y}_{2}}{\partial \lambda}\right)+\mathrm{G}_{1}[\phi, \psi] \frac{\partial \mathrm{y}_{1}}{\partial \lambda}+\mathrm{G}_{2}[\phi, \psi] \frac{\partial \mathrm{y}_{2}}{\partial \lambda}
\end{aligned}
$$

Under $\lambda=0$, and replacing $\left[y_{1}, y_{2}\right]$ by $\left[y_{1}^{*}, y_{2}^{*}\right]$, equation (3.9) by equality (3.8) becomes

$$
\begin{aligned}
& \frac{d \phi}{d z}(z ; c)=\frac{d^{2}}{d z^{2}}\left(\frac{\partial y_{1}^{*}}{\partial \lambda}\right)-c \frac{d}{d z}\left(\frac{\partial y_{1}^{*}}{\partial \lambda}\right)+F_{1}[\phi, \psi] \frac{\partial y_{1}^{*}}{\partial \lambda}+F_{2}[\phi, \psi] \frac{\partial y_{2}^{*}}{\partial \lambda}, \\
& \frac{d \psi}{d z}(z ; c)=-c \frac{d}{d z}\left(\frac{\partial y_{2}^{*}}{\partial \lambda}\right)+G_{1}[\phi, \psi] \frac{\partial y_{1}^{*}}{\partial \lambda}+G_{2}[\phi, \psi] \frac{\partial y_{2}^{*}}{\partial \lambda},
\end{aligned}
$$

where $\frac{\partial y_{i}^{*}}{\partial \lambda}=\frac{\partial y_{i}^{*}}{\partial \lambda}(z ; 0, c)$ now. On the other hand, differentiating equation $(2.1)$ with respect to $c$, we get 


$$
\begin{aligned}
& \frac{d^{2}}{d z^{2}}\left(\frac{\partial \phi}{\partial c}\right)-\frac{d \phi}{d z}-c \frac{d}{d z}\left(\frac{\partial \phi}{\partial c}\right)+F_{1}[\phi, \psi] \frac{\partial \phi}{\partial c}+F_{2}[\phi, \psi] \frac{\partial \psi}{\partial c}=0, \\
& -\frac{d \psi}{d z}-c \frac{d}{d z}\left(\frac{\partial \psi}{\partial c}\right)+G_{1}[\phi, \psi] \frac{\partial \phi}{\partial c}+G_{2}[\phi, \psi] \frac{\partial \psi}{\partial c}=0,
\end{aligned}
$$

where $\phi=\phi(z ; c)$ and $\psi=\psi(z ; c)$. Therefore both $\left[\frac{\partial y_{1}^{*}}{\partial \lambda}(z ; 0 ; c), \frac{\partial y_{2}^{*}}{\partial \lambda}(z ; 0, c)\right]$ and $\left[\frac{\partial \phi}{\partial c}(z ; c), \frac{\partial \psi}{\partial c}(z ; c)\right]$ satisfy the same differential equation. In addition, differentiation of the initial condition (3.7) yields

$$
\underline{v_{\lambda}}(0 ; \lambda, c)=\underline{w_{c}}(0 ; c) \text {, }
$$

in particular,

$$
v_{\lambda}(0 ; 0, c)=\underline{w_{c}}(0 ; c)
$$

and hence the equality

$$
\underline{v_{\lambda}^{*}}(z ; 0, c)=\underline{w}_{c}(z ; c), 0 \leq z \leq p(c) \text {. }
$$

The equalities (3.8) and (3.12) together give

$$
\begin{gathered}
\underline{\mathrm{v}}^{*}(\mathrm{z} ; \lambda, \mathrm{c})=\underline{\mathrm{w}}_{\mathrm{z}}(\mathrm{z} ; \mathrm{c})+\lambda \underline{\mathrm{w}_{\mathrm{c}}}(\mathrm{z} ; \mathrm{c})+0\left(\lambda^{2}\right), \\
0 \leq \mathrm{z} \leq \mathrm{p}(\mathrm{c}), \text { as } \lambda \rightarrow 0
\end{gathered}
$$

Knowing $\underline{v}^{*}(z ; \lambda, c)=x(z ; \lambda, c) \underline{v}^{*}(0 ; \lambda, c)$, by equation (3.13) for $z=p(c)$ and a1so $z=0$, we get

$$
\begin{aligned}
& \underline{w_{z}}(p(c) ; c)+\lambda \underline{w_{c}}(p(c) ; c)+0\left(\lambda^{2}\right) \\
& \left.=X(p(c) ; \lambda, c) \underline{w_{z}}(o ; c)+\lambda \underline{w_{c}}(0 ; c)\right] .
\end{aligned}
$$

Substitution of the equation (3.6) containing $p^{\prime}(c)$ into the left hand side of equation (3.14) and periodicity lead to

$$
\begin{aligned}
& X(p(c) ; \lambda, c)\left[w_{z}(0 ; c)+\lambda \underline{w_{c}}(0 ; c)\right] \\
& =\left[1-\lambda p^{\prime}(c)\right]\left[w_{z}(0 ; c)+\lambda w_{c}(0 ; c)\right]+0\left(\lambda^{2}\right) .
\end{aligned}
$$

Hence the eigenvalue $\mu_{1}(\lambda, c)$ satisfies

$$
\left.\frac{\partial}{\partial \lambda} \mu_{1}(\lambda, c)\right|_{\lambda=0}=-p^{\prime}(c)
$$

The proof is now complete. 
On the other hand, under certain conditions, two eigenvalues have modulus less than one and one has modulus greater than one.

THEOREM 3.3. Suppose $(a) F_{2}(u, w)$ is a non-zero constant and (b) $G_{1}(u, w)$ and $G_{2}(u, w)$ are constant, then for $\lambda$ sufficiently large, two eigenvalues of $X(p(c) ; \lambda, c)$ have modulus $<1$ and one has modulus $>1$.

PROOF: Decompose the matrix $\mathrm{A}(z ; \lambda, \mathrm{c})$ as follows

$A(z ; \lambda, c)=B(\lambda, c)+E(z ; c)$

$$
=\left[\begin{array}{ccc}
0 & 1 & 0 \\
\lambda & c & -F_{2} \\
\frac{G_{1}}{c} & 0 & \frac{G_{2}^{-\lambda}}{c}
\end{array}\right]+\left[\begin{array}{lll}
0 & 0 & 0 \\
-F_{1}[\phi(z ; c), \psi(z ; c)] & 0 & 0 \\
0 & 0 & 0
\end{array}\right] \text {. }
$$

Let $s_{i}(\lambda, c), i=1,2,3$ be the eigenvalues of $B(\lambda, c)$ and $q_{i}$ the corresponding eigenvectors. The characteristic equation of $B(\lambda, c)$ is

$$
-s^{3}+\left(\frac{G_{2}-\lambda}{c}+c\right) s^{2}+\left(2 \lambda-G_{2}\right) s+\lambda\left(\frac{\lambda-G_{2}}{c}\right)-\frac{F_{2} G_{1}}{c}=0 .
$$

It follows that as $\lambda+\infty$,

$$
\begin{aligned}
& s_{1}(\lambda, c)=\frac{-\lambda}{c}+0(1) \\
& s_{2}(\lambda, c)=-\sqrt{\lambda}+0(1) \\
& s_{3}(\lambda, c)=\sqrt{\lambda}+0(1) .
\end{aligned}
$$

The vectors $q_{i}(\lambda, c)$ are

$$
\underline{q_{i}}(\lambda, c)=\left[\begin{array}{l}
1 \\
s_{i} \\
\frac{s_{i}^{2}-c s_{i}-\lambda}{-F_{2}}
\end{array}\right], i=1,2,3
$$

and let $Q(\lambda, c)$ be the non-singular matrix

$$
Q(\lambda, c)=\left[\underline{q_{1}}(\lambda, c), \underline{q_{2}}(\lambda, c), \underline{q_{3}}(\lambda, c)\right],
$$

then

$$
Q^{-1} \mathrm{BQ}=\left[\begin{array}{ccc}
\mathrm{s}_{1}(\lambda, \mathrm{c}) & 0 & 0 \\
0 & \mathrm{~s}_{2}(\lambda, v) & 0 \\
0 & 0 & \mathrm{~s}_{3}(\lambda, c)
\end{array}\right] \text {. }
$$


Now consider the matrix

$$
\mathrm{Y}(\mathrm{z} ; \lambda, \mathrm{c})=\mathrm{Q}^{-1} \mathrm{X}(\mathrm{z} ; \lambda, \mathrm{c}) \mathrm{Q}
$$

which has the same eigenvalues as $X(z ; \lambda, c)$, in particular with $z=p(c)$, and satisfies the differential equation

$$
\begin{aligned}
\frac{d}{d z} Y(z ; \lambda, c) & =Q^{-1} A(z ; \lambda, c) Q Y(z ; \lambda, c) \\
& =\left[Q^{-1} B(\lambda, c) Q+Q^{-1} E(z ; c) Q\right] Y(z ; \lambda, c),
\end{aligned}
$$

since $\frac{d}{d z} X(z ; \lambda, c)=A(z ; \lambda, c) X(z ; \lambda, c)$.

But $Q^{-1} \mathrm{BQ}$ is the diagonal matrix from before and it can be shown easily using (3.15) and (3.16) that all elements of $Q^{-1} E Q$ are $o(1)$ as $\lambda \rightarrow \infty$, therefore the eigenvalues of $\mathrm{Y}(\mathrm{p}(\mathrm{c}) ; \lambda, \mathrm{c})$ and hence of $\mathrm{X}(\mathrm{p}(\mathrm{c}) ; \lambda, \mathrm{c})$ approach

$$
\exp \left[s_{i}(\lambda, c) p(c)\right], i=1,2,3 \text { as } \lambda \rightarrow \infty \text {. }
$$

It follows from (3.15) that as $\lambda \rightarrow \infty$, two eigenvalues of $X(p(c) ; \lambda, c)$ have modulus $<1$ and one has modulus $>1$.

To summarize, under the assumptions of both Theorem (3.2) and Theorem (3.3), at least two eigenvalues of $\mathrm{X}(\mathrm{p}(\mathrm{c}) ; \lambda, c)$ have modulus $>1$ as $\lambda \rightarrow 0+$, and two eigenvalues of $X(p(c) ; \lambda, c)$ have modulus $<1$ as $\lambda \rightarrow \infty$. Hence one of the eigenvalues must have modulus $=1$ for some $\lambda>0$ and under Theorem (3.1), the travelling wave solution $(\phi(z ; c), \psi(z ; c))$ is unstable.

\section{REFERENCES}

1. FITzhuGH, R. Mathematical Models of Excitation and Propagation in Nerve, in Biologica1 Engineering, McGraw-Hi11 (1969), 1-85.

2. Nagumo, J, ARImoto, S., and Yoshizawa, S. An Active Pulse Transmission Line Simulating Nerve Axon, Proc. I.R.E. 50 (1962), 2061.

3. ALLEN, Linda J.S. Persistence and Extinction in Lotka-Volterra Reaction-Diffusion Equations, Mathematical BioSciences 65 (1983), 1.

4. COSNER, C. Pointwise a Priori Bounds for Strongly Coupled Semilinear Systems of Parabolic Partial Differential Equations, Indiana University Mathematics Journal 30 (1981), 607 .

5. GARDNER, R.A. Asymptotic Behavior of Semilinear Reaction-Diffusion Systems with Dirichlet Boundary Conditions, Indiana University Mathematics Journal 29 (1980), 161 .

6. GURTIN, M.E. and MAC CAMY, R.C. Product Solutions and Asymptotic Behavior for Age-Dependent, Dispersing Populations, Mathematical Biosciences 62 (1982), 157.

7. JACQUEZ, J.A. The Physiological Role of Myoglobin: More than a Problem in Reaction-Diffusion Kinetics, Mathematical Biosciences 68 (1984), 57. 
8. LEUNG, A. A Semiliner Reaction - Diffusion Prey-Predator System with Nonlinear Coupled Boundary Conditions: Equilibrium and Stability, Indiana University Math. J. 31 (1982), 223.

9. SCHATZMan, M. Stationary Solutions and Asymptotic Behavior of a Quasilinear Degenerate Parabolic Equation, Indiana University Math. J. 33 (1984), 1 .

10. EVANS, J.H. Nerve Axon Equations: IV. The Stable and The Unstable Impulse, Indiana University Math. J. 24 (1975), 1169.

11. HASTINGS, S. The Existence of Periodic Solutions to Nagumo's Equation, Quart. J. Math. $25(1974), 369$.

12. RINZEL, J. and KELLER, J.B. Travelling Wave Solutions of a Nerve Conduction Equation, Biophys. J. 13 (1973), 1313.

13. DAI, L.S. A Stable Travelling-Wave Solution of a Model of Cellular Control Processes with Positive Feedback, Mathematical Biosciences 61 (1982), 267. 


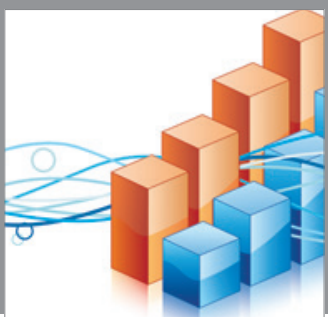

Advances in

Operations Research

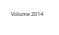

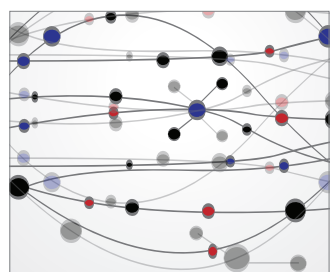

\section{The Scientific} World Journal
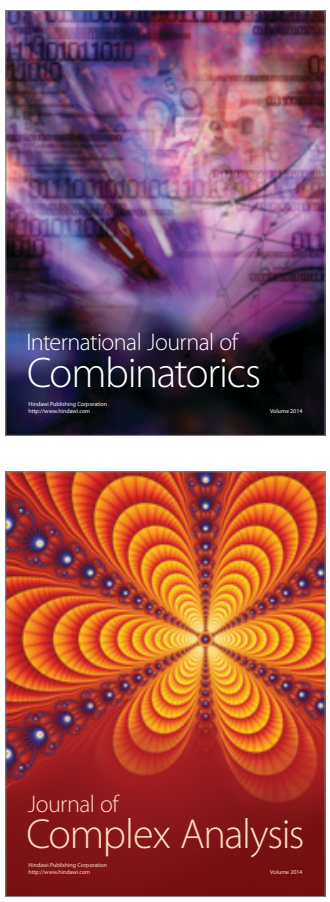

International Journal of

Mathematics and

Mathematical

Sciences
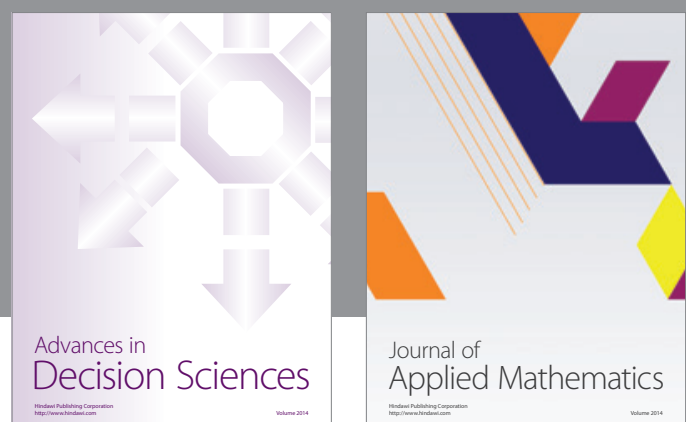

Journal of

Applied Mathematics
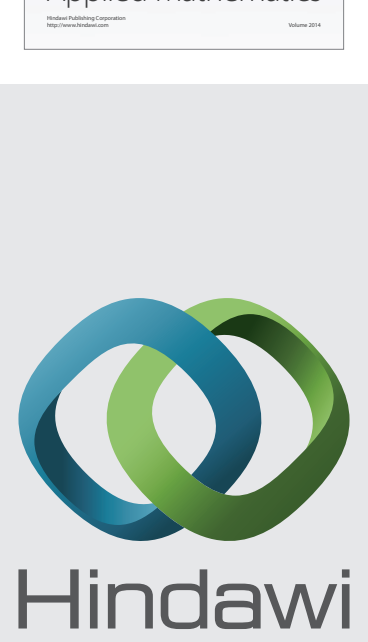

Submit your manuscripts at http://www.hindawi.com
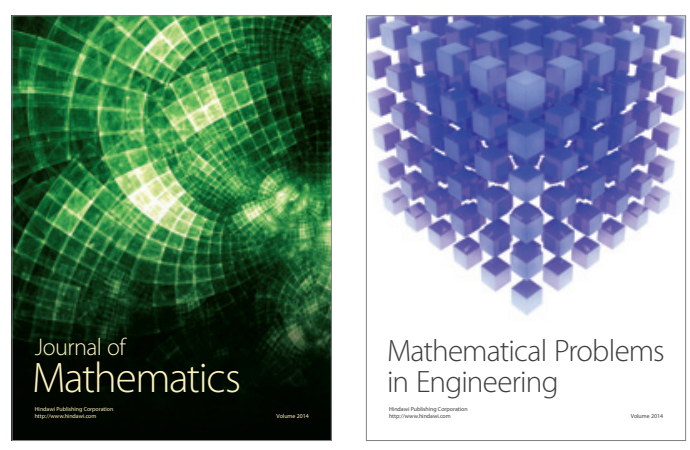

Mathematical Problems in Engineering
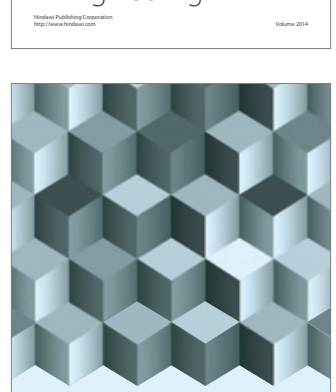

Journal of

Function Spaces
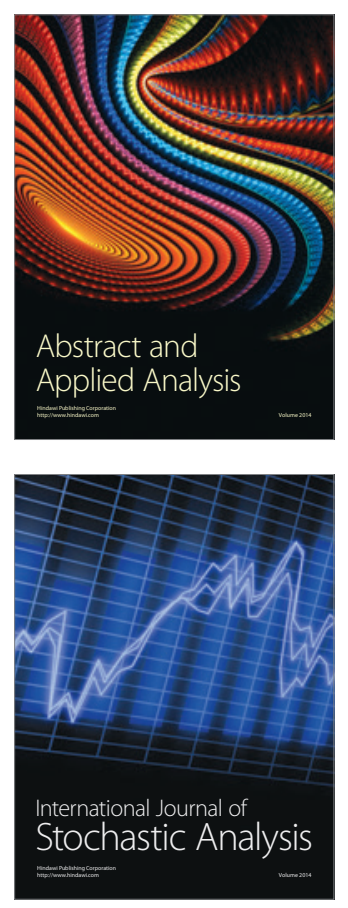

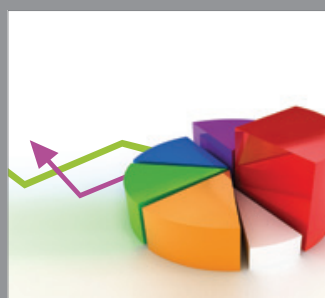

ournal of

Probability and Statistics

Promensencen
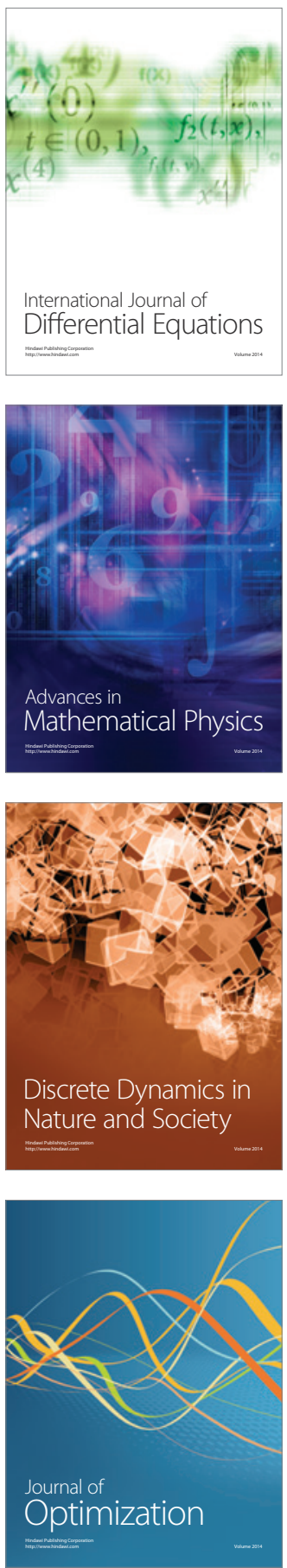